\title{
Cost-Effectiveness Analysis of Cardiovascular Disease Treatment in Japan
}

\author{
Satoshi Kodera, ${ }^{1} \mathrm{MD}$, Arihiro Kiyosue, ${ }^{1} \mathrm{MD}$, Jiro Ando, ${ }^{1} \mathrm{MD}$, Hiroshi Akazawa, ${ }^{1} \mathrm{MD}$, \\ Hiroyuki Morita, ${ }^{1}$ MD, Masafumi Watanabe, ${ }^{1}$ MD and Issei Komuro, ${ }^{1}$ MD
}

\begin{abstract}
Summary
The quality-adjusted life year (QALY) and incremental cost-effectiveness ratio (ICER) are important concepts in cost-effectiveness analysis, which is becoming increasingly important in Japan. QALY is used to estimate quality of life (QOL) and life years, and can be used to compare the efficacies of cancer and cardiovascular treatments. ICER is defined as the difference in cost between treatments divided by the difference in their effects, with a smaller ICER indicating better cost-effectiveness. Here, we present a review of cost-effectiveness analyses in Japan as well other countries. A number of treatments were shown to be cost-effective, e.g., statin for secondary prevention of cardiovascular disease, aspirin for primary prevention of cardiovascular disease, DOAC for high-risk atrial fibrillation, beta blockers, ACE inhibitors, and ARB for heart failure, sildenafil and bosentan for pulmonary hypertension, CABG for multi-vessel coronary disease, ICD for ventricular tachycardia, and CRT for heart failure with low ejection fraction, while others were not cost-effective, e.g., epoprostenol for pulmonary hypertension and LVAD for end-stage heart failure. Further investigations are required regarding some treatments, e.g., PCSK-9 inhibitors for familial hypercholesterolemia, PCI for multi-vessel coronary disease, catheter ablation for atrial fibrillation, and TAVI for severe aortic stenosis. Ethical aspects should be taken into consideration when utilizing the results of cost-effectiveness analysis in medical policy.
\end{abstract}

(Int Heart J 2017; 58: 847-852)

Key words: ICER, Heart, QALY, Japanese

$\mathrm{M}$ edical costs are increasing in developed countries, including Japan, with the aging of the population and advances in technology. Although several drugs and devices have improved clinical outcomes, the increases in medical costs associated with these expensive options are placing pressure on national budgets. Efficient usage of medical budgets is crucial for maintenance of a sustainable medical system. Costeffectiveness analysis was developed to make efficient use of medical budgets. ${ }^{1)}$ Cost-effectiveness analysis evaluates not only the monetary costs of drugs or procedures, but also takes into account their effects on quality of life (QOL). Several countries, including the UK and Australia, introduced cost-effectiveness analysis to approve new drugs, ${ }^{2)}$ and Japan is planning to introduce costeffectiveness analysis for health policy in 2018. Costeffectiveness analysis is becoming increasingly important all around the world. Guidelines for the development, reporting, and evaluation of health care systems recommend the inclusion of cost-effectiveness analysis. ${ }^{3)}$ To understand cost-effectiveness analysis, it is necessary to understand key concepts, such as quality adjusted life year (QALY) or the incremental cost-effectiveness ratio (ICER). In the first part of this review, we will introduce key concepts. In the second part, we will summarize costeffectiveness data for cardiology drugs and cardiology devices. We have reviewed studies conducted in Japan as well as in other countries.

\section{Key Concepts of Cost-Effectiveness Analysis}

Definition of cost: Two types of cost are taken into consideration in cost-effectiveness analysis, i.e., direct costs and indirect costs. ${ }^{4)}$ Direct costs are all costs of resources used that are attributable to a health care intervention, and can be divided into direct medical costs and direct nonmedical costs. Direct medical costs are the costs of inpatient and outpatient treatment, while direct non-medical costs are payments other than those for the medical institute, e.g., the costs of transportation to the hospital. Indirect costs represent opportunity loss, e.g., the loss of working opportunity because of disease. When performing cost-effectiveness analysis, we estimate the costs using real calculated costs, previously published data, or national data.

Analysis: Clarification of the analysis standpoint is necessary in cost-effectiveness analysis. ${ }^{5)}$ The Consolidated Health Economic Evaluation Reporting Standards

From the ${ }^{1}$ Department of Cardiovascular Medicine, Graduate School of Medicine, The University of Tokyo, Tokyo, Japan.

Address for correspondence: Satoshi Kodera, MD, Department of Cardiovascular Medicine, Graduate School of Medicine, The University of Tokyo, 7-3-1 Hongo, Bunkyo-ku, Tokyo 113-8655, Japan. E-mail: kodera@tke.att.ne.jp

Received for publication June 30, 2017. Revised and accepted July 26, 2017.

Released in advance online on J-STAGE November 17, 2017.

doi: 10.1536/ihj.17-365

All rights reserved by the International Heart Journal Association. 


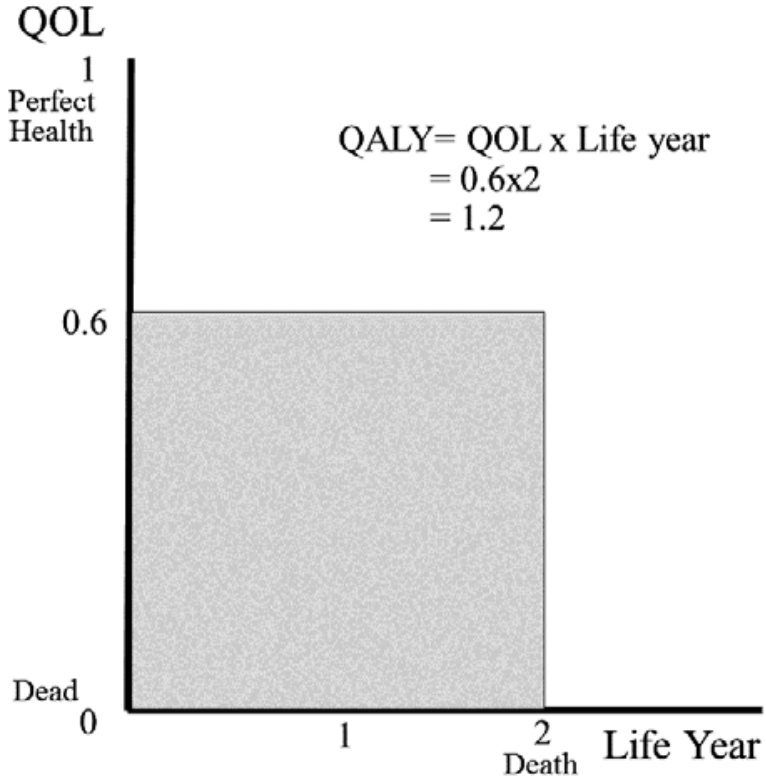

Figure 1. Concept of quality of life (QOL) adjusted life year (QALY). For a patient with QOL 0.6 who lives for 2 years, QALY is $0.6 \times 2=1.2$.

(CHEERS) statement and Japanese guidelines recommend clarification of the analysis standpoint, ${ }^{6,7)}$ e.g., patient, medical system, and social. From the patient standpoint, costs include hospital payment, drug payment, transportation fees, and loss of working opportunity. From the standpoint of the medical system, costs include patient payments, insurance payments, and government payments. From the social standpoint, costs include patient payments, insurance payments, government costs, and opportunity loss. Most cost-effectiveness analyses use the medical system or social standpoint.

Utility: Utility is defined as the expected value. Quality of life (QOL) includes physical value and psychological value, and is a useful parameter to evaluate the utility of a medical treatment. QOL evaluation can be divided into the profile type and the estimate type. ${ }^{8)}$ Profile type QOL, such as SF-36, evaluates health status in detail based on sufficient amounts of specific information regarding health and QOL. Estimate type QOL, such as EQ-5D, is often used in cost-effectiveness analysis. QOL 1.0 indicates perfect condition, while QOL 0 indicates death.

QALY: Quality-adjusted life year (QALY) is a parameter used to estimate QOL and life year gain,, and is calculated as: QALY $=$ QOL $\times$ Life year gain. Figure 1 shows the concept of QALY. For example, if a patient has QOL 0.6 and lives for 2 years, QALY is $0.6 \times 2=1.2$. QALY can be used to estimate QOL and life expectancy, and is a useful parameter to evaluate treatment efficacy. For example, QALY can be used to compare the efficacies of cancer treatment and cardiovascular treatment.

Definition of ICER: The incremental cost-effectiveness ratio (ICER) is an important parameter used for comparison of treatment efficacy, ${ }^{4)}$ and is defined as the difference in cost between treatments divided by the difference in effect. When comparing the cost-effectiveness of treatment

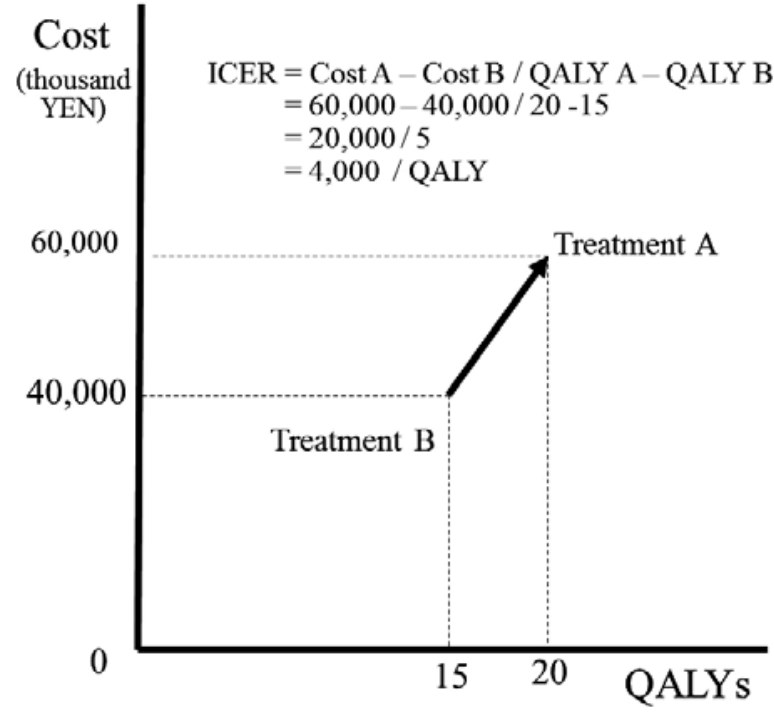

Figure 2. Estimation of incremental cost-effectiveness ratio (ICER). If the cost of treatment A is 60,000,000 yen and the cost of treatment B is $40,000,000$ yen, the quality of life adjusted life year (QALY) of treatment A is 20 QALYs and that of treatment B is 15 QALYs. The ICER of treatment A over treatment B is 4,000,000 yen/QALY.

$\mathrm{A}$ and treatment $\mathrm{B}, \mathrm{ICER}=($ Cost $\mathrm{A}-$ Cost $\mathrm{B}) /($ Utility $\mathrm{A}-$ Utility B). Figure 2 shows the estimation of ICER. If the cost of treatment $\mathrm{A}$ is $60,000,000$ yen and the cost of treatment B is 40,000,000 yen, the QALYs of treatments $\mathrm{A}$ and $\mathrm{B}$ are 20 and 15 QALYs, respectively. The ICER of treatment A over treatment B is 4,000,000 yen/QALY. If the incremental cost is small or the incremental effect is large, ICER would be small. Therefore, a smaller ICER indicates better cost-effectiveness. ICER can be calculated by QALY and also life year. The Japanese guidelines recommend using ICER in cost-effectiveness analysis.7)

Cost-effectiveness threshold: The cost-effectiveness threshold is used to evaluate whether treatments are good or very good value for money. ${ }^{4)}$ Willing to pay (WTP) is a cost-effectiveness threshold of payment for treatment. ${ }^{10)}$ WTP is estimated using a questionnaire and is useful to evaluate whether ICER is cost-effective. The UK has ICER criteria of $£ 20,000$ - $£ 30,000$ British pounds (GBP) per QALY. ${ }^{11)}$ The cost-effectiveness of treatment A is good if its ICER over treatment B is less than GBP $£ 20,000$. Conversely, treatment A shows poor cost-effectiveness if its ICER over treatment B is more than GBP 330,000 . The WTP in the United States is around \$50,000$\$ 100,000$ per QALY, ${ }^{12)}$ and that in Japan is around 5,000,000-6,000,000 yen per QALY. ${ }^{12)}$ In Japan, treatment A would be considered to have good cost-effectiveness if its ICER over treatment B is less than 5,000,000 yen per QALY. In the case of life year gain, the threshold of costeffectiveness would be 4,000,000-5,000,000 yen per life year in Japan.

Health economic analysis: The most common type of health economic analysis is cost-effectiveness analysis, which is a research method applied to estimate the incremental benefits and costs of a new treatment compared with the standard treatment. ${ }^{4)}$ Cost-effectiveness analysis is 


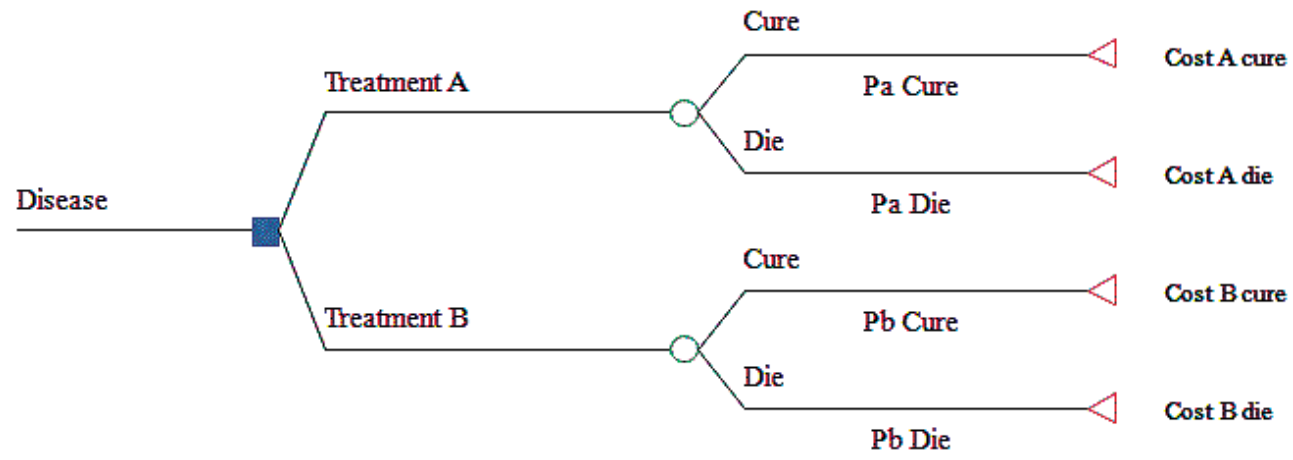

Figure 3. Decision tree model. The decision tree includes all treatment choices and results of the treatments. The probability of cure with treatment $\mathrm{A}$ is PaCure. To compare the cost-effectiveness of treatments $\mathrm{A}$ and $\mathrm{B}$, we calculate $($ PaCure $\times$ Cost A cure + PaDie $\times$ Cost $\mathrm{A}$ die $) /($ PaCure $\times \mathrm{QOL})$ versus $(\mathrm{PbCure} \times$ Cost $\mathrm{B}$ cure $+\mathrm{PbDie} \times$ Cost $\mathrm{B}$ die $) /$ $(\mathrm{PbCure} \times \mathrm{QOL})$.

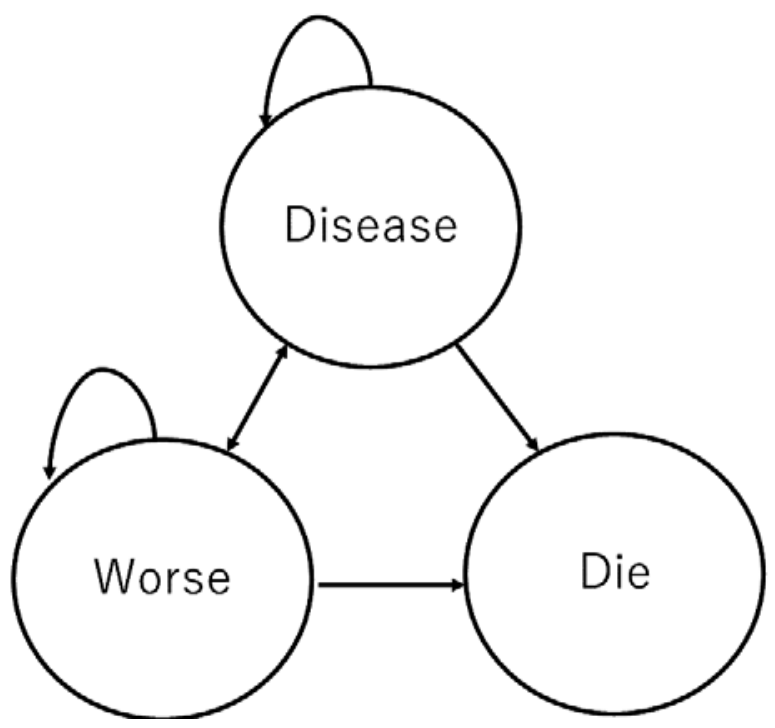

Figure 4. Markov model. The Markov model contains state transition probabilities.

expressed in terms of ICER, and the most commonly used outcome measure is QALY. Cost-effectiveness analysis is often presented on a 4-quadrant plane, with cost represented on the $\mathrm{x}$-axis and effectiveness on the y-axis. Clinical trials are performed to estimate clinical effects, and a randomized control trial or meta-analysis of clinical trials is recommended for use of health economic analysis. ${ }^{7)}$

Analysis model and time horizon: Cost-effectiveness analysis is performed using two types of model, i.e., decision tree models and Markov models. ${ }^{4)}$ The decision tree model is suitable for an acute disease, and includes all treatment choices and results of treatments (Figure 3). A Markov model is suitable for a chronic disease, and is useful when the event examined has occurred repeatedly (Figure 4). When performing cost-effectiveness analysis, it is necessary to take into account long-term effects and costs. When limited data are available from published clinical trials, it is necessary to estimate the results over a longer time frame; therefore, a model capable of providing estimates over a longer time is required. A Markov model contains state transition probabilities, and can be used to calculate long-term cost and effectiveness. The time horizon, i.e., the analysis period, should be long enough to estimate the effects of the treatment.

Sensitivity analysis: Cost-effectiveness analysis requires a number of parameters to be defined (cost, utility, event rate, etc.), and these parameters contain uncertainties. Sensitivity analysis, which is a statistical method to evaluate the robustness of results when parameters contain uncertainty, ${ }^{4)}$ is therefore necessary. In sensitivity analysis, the parameters are adjusted from the lowest to the highest number.

Medical ethics: Ethical aspects should be taken into consideration when utilizing the results of cost-effectiveness analysis in medical policy. Medical policy should take into account the following 3 ethical values: treating people equally, favoring the worse-off, and maximizing total benefits. ${ }^{13)}$ However, cost-effectiveness analysis only considers maximizing total benefits. The cost-effectiveness of device therapy for end-stage heart failure would be worse than use of sildenafil for erectile dysfunction. ${ }^{14)}$ However, this contravenes the ethical consideration of favoring the worse-off. Those with short life expectancy may achieve only little QALY with treatment. ICER of terminal care would be worse than pediatric treatments. In addition, ICER of expensive therapies for rare diseases would be worse than common disease treatments. This would mean that terminal patients and those with rarer diseases would not be treated equally. These examples indicate that costeffectiveness analysis is insufficient for evaluating medical treatment in medical policy decision making.

\section{Specific Cost-Effectiveness Analysis in the Field of Cardiology}

Dyslipidemia: A Japanese study showed that the ICER of statin for primary prevention of cardiovascular disease compared with conventional therapy was 12,690,000$329,460,000$ yen per QALY, ${ }^{15)}$ which suggested that primary prevention with statin was not cost-effective in Japan. A study performed in the United States indicated the ICERs of statins in primary prevention of cardiovascular disease for patients at high, intermediate, and low risk 
were $\$ 37,000, \$ 81,000$, and $\$ 140,000$ per QALY, respectively. ${ }^{16)}$ These observations suggested that statins were cost-effective in primary prevention of cardiovascular disease for high-risk patients but not for low-risk patients.

The ICERs of protein convertase subtilisin/kexin type 9 (PCSK9) inhibitors over ezetimibe were reported to be $\$ 503,000$ per QALY for heterozygous familial hypercholesterolemia and \$414,000 per QALY for atherosclerotic cardiovascular disease in a study performed in the United States. ${ }^{17)}$ This study suggested that PCSK9 inhibitors are not cost-effective in the United States. However, there have been no Japanese studies regarding the costeffectiveness of PCSK9 inhibitors.

Antithrombotic agents: A review article showed that aspirin was dominant in primary prevention of cardiovascular disease over no medication (less cost and more effect). ${ }^{18)}$ Adding aspirin improved QALY and reduced cost by reducing cardiovascular events. A Japanese study also showed dominance of aspirin over no medication in primary prevention of cardiovascular disease. ${ }^{19)}$ These data indicated that aspirin has very good cost-effectiveness in cardiovascular primary prevention all around the world. A study in the United States indicated that the ICER of clopidogrel over aspirin for secondary prevention of cardiovascular disease was more than $\$ 130,000$ per QALY. $^{20)}$ This study suggested that clopidogrel was not costeffective for secondary prevention in the United States. A Canadian study showed an ICER of $\$ 57,630$ per QALY for prasugrel over clopidogrel. ${ }^{21)}$ In a study in Japan, the ICER of cilostazol over aspirin for secondary prevention of cerebral infarction was 1,800,000 yen per QLAY, ${ }^{22}$ which suggested that cilostazol was cost-effective for secondary prevention of cerebral infarction in Japan.

A meta-analysis examining the cost-effectiveness of direct oral anticoagulant (DOAC) use for stroke prevention in atrial fibrillation indicated that DOAC was costeffective over warfarin. ${ }^{23)}$ The ICER of rivaroxaban over warfarin was $\$ 3,190-\$ 55,757$ per QALY, that of dabigatran over warfarin was $\$ 11,150-\$ 20,797$ per QALY, and that of apixaban showed a gain of $\$ 4,723-\$ 24,312$ per QALY over warfarin. In a Japanese study, the ICER of apixaban over warfarin for atrial fibrillation was $\$ 22,500$ per QALY. ${ }^{24)}$ These $^{4)}$ data indicated that although DOACs were expensive, they showed acceptable cost-effectiveness for stroke prevention in atrial fibrillation.

Heart failure treatment:

Beta blockers: A study performed in the United States indicated an ICER of beta-blocker over no beta-blocker of $\$ 7,043$ per life year gain in patients with heart failure. ${ }^{25)}$ In a study performed in the United Kingdom, carvedilol was shown to have a lower cost and better clinical effect over conventional therapy in patients with severe heart failure. ${ }^{26)}$ A Japanese study showed that the 5-year costs of carvedilol versus conventional therapy were 3,500,000 yen versus 5,500,000 yen, respectively, and life expectancy with carvedilol versus conventional therapy was 121 months versus 88 months, respectively. This study showed that carvedilol had a lower cost and provided longer life expectancy than conventional therapy for chronic heart failure. ${ }^{27)}$ These data showed that beta-blockers have good cost-effectiveness in heart failure patients all around the world.

Angiotensin converting enzyme (ACE) inhibitor, angiotensin receptor blocker (ARB), and others: A study performed in the United States showed that the ICER of captopril over placebo was $\$ 3,700-\$ 10,400$ per QALY after myocardial infarction. ${ }^{28)}$ In another American study, the ICER of enalapril over placebo was $\$ 115$ per QALY for symptomatic heart failure. ${ }^{29)}$ A German study indicated an ICER of $\$ 1,700-\$ 5,800$ per life year gain for ramipril over placebo for heart failure after myocardial infarction. ${ }^{30}$ A study performed in the United States showed that valsartan was dominant over placebo for chronic heart failure (less cost and better effect), ${ }^{31)}$ and a British study showed that candesartan was dominant over placebo for heart failure (less cost and better effect). ${ }^{32}$ These data indicated that ACE inhibitors and ARBs are cost-effective for the treatment of heart failure in various countries. A Japanese study showed tolvaptan reduced the cost of treatment for heart failure in aquaporin-defined responders. ${ }^{33)}$ Compared with conventional therapy, tolvaptan showed lower medical cost (1,120,000 yen/year versus 3,120,000 yen/year, respectively) and shorter hospital stay (30 days versus 70 days, respectively). Tolvaptan could be cost-effective in certain types of heart failure.

Drugs for pulmonary hypertension: In a British study, the ICERs of epoprostenol, iloprost, and bosentan over supportive care for functional class 3 pulmonary hypertension were GBP£277,000, GBP£101,000, and GBP $£ 27,000$ per QALY, respectively, and sildenafil was dominant (less cost and better effect). ${ }^{34)}$ These data suggested that bosentan and sildenafil are cost-effective treatments for pulmonary hypertension, while epoprostenol is not. This is consistent with network meta-analysis indicating that bosentan and sildenafil had superior effectiveness over other oral medications for pulmonary artery hypertension. ${ }^{35)}$ To date, there have been no cost-effectiveness analyses of pulmonary hypertension drugs in Japan.

Percutaneous coronary intervention (PCI) and coronary artery bypass grafting (CABG): A study performed in the United States showed an ICER of $\$ 6,791$ per QALY for coronary artery bypass graft (CABG) over percutaneous coronary intervention (PCI) with drugeluting stents in diabetic multivessel disease. ${ }^{36)}$ The Synergy between percutaneous coronary intervention with TAXUS and cardiac surgery (SYNTAX) trial showed an ICER of CABG over PCI of $\$ 16,537$ per QALY in left main coronary disease or 3-vessel disease. ${ }^{37}$ A metaanalysis to examine the cost-effectiveness of PCI versus CABG indicated a favorable ICER for CABG rather than PCI in multivessel coronary artery disease (CAD). ${ }^{38)} \mathrm{A}$ prospective observational study performed in the United Kingdom reported an ICER of GBP $£ 47,000$ per QALY for PCI over medical treatment in angina pectoris, ${ }^{39)}$ and a Japanese study indicated an ICER of PCI over medical therapy in angina patients of $\$ 7,000$ per QALY. ${ }^{40)}$ Drugeluting stents showed better cost-effectiveness than bare metal stents in Japan. ${ }^{41)}$ These data suggested that PCI is more cost-effective than medical therapy for angina in Japan. Meta-analysis suggested that CABG would have better cost-effectiveness than PCI for multivessel disease in Japan as well as other countries. ${ }^{38)}$ 
Catheter ablation: A meta-analysis indicated that catheter ablation was associated with high costs and high rates of complications. ${ }^{42)}$ No evidence is available regarding important variables in ablation, and it is difficult to evaluate its cost-effectiveness. However, a Japanese analysis showed an ICER of ablation plus warfarin over warfarin of 1,692,399-2,747,037 yen per QALY for atrial fibrillation with $\mathrm{CHADS}_{2}$ score 4-6. These data indicated that ablation was cost-effective for high-risk patients. ${ }^{43}$

Implantable cardioverter defibrillator (ICD) and cardiac resynchronization therapy (CRT): A meta-analysis and systematic review performed in the United Kingdom reported an ICER of GBP£14,231-GBP£29,756 per QALY for implantable cardioverter defibrillators (ICD) compared with optimal medical therapy, ${ }^{44)}$ and the ICER of cardiac resynchronization therapy (CRT) compared with optimal therapy was GBP £27,584-GBP £27,899 per QALY. These data indicated that both ICD and CRT represent cost-effective treatments. However, there have been no cost-effectiveness analyses of ICD and CRT in Japan.

Transcatheter aortic valve implantation (TAVI): A meta-analysis showed controversial results of transcatheter aortic valve implantation (TAVI) ICER over surgical aortic valve replacement (AVR) for severe aortic stenosis. ${ }^{45)}$ Two of 7 studies indicated that AVR was dominant (less cost and better effect), two studies showed that TAVI was dominant (less cost and better effect), and 3 studies showed ICERs of $\$ 32,000-\$ 1,014,949$ per QALY for TAVI over AVR in severe aortic stenosis. Thus, further cost-effectiveness analyses of TAVI are required.

Left ventricular assist device (LVAD): In a British study, the ICER of left ventricular assist devices (LVAD) over conventional therapy for end-stage heart failure was GBP $£ 79,212$ per QALY. ${ }^{46)}$ Another meta-analysis showed an ICER of $\$ 89,790-\$ 414,274$ per QALY for LVAD over heart transplantation for end-stage heart failure. ${ }^{47)}$ These data suggested that LVAD is not cost-effective for endstage heart failure. All reports regarding the ICER of LVAD published to date were based on retrospective observational studies, and estimation of the costeffectiveness may not be accurate. To date, there have been no Japanese studies regarding ICER of LVAD.

\section{Conclusions}

While some treatments are cost-effective (statins for secondary prevention of cardiovascular disease, aspirin for primary prevention of cardiovascular disease, DOAC for high-risk atrial fibrillation, beta blockers, ACE inhibitors, and ARB for heart failure, sildenafil and bosentan for pulmonary hypertension, CABG for multivessel coronary disease, ICD for ventricular tachycardia, CRT for severe heart failure with low ejection fraction), others are not (epoprostenol for pulmonary hypertension, LVAD for endstage heart failure). Further investigations are required regarding some treatments (PCSK-9 inhibitors for familial hypercholesterolemia, PCI for multivessel coronary disease, catheter ablation for atrial fibrillation, TAVI for severe aortic stenosis). Ethical aspects should be taken into consideration when utilizing the results of costeffectiveness analysis in medical policy.

\section{Disclosures}

Conflicts of interest: The authors declare that they have no conflicts of interest.

\section{References}

1. Weinstein MC, Skinner JA. Comparative effectiveness and health care spending-implications for reform. N Engl J Med 2010; 362: 460-5.

2. Clement FM, Harris A, Li JJ, Yong K, Lee KM, Manns BJ. Using effectiveness and cost-effectiveness to make drug coverage decisions: a comparison of Britain, Australia, and Canada. JAMA 2009; 302: 1437-43.

3. Brouwers MC, Kho ME, Browman GP, et al. AGREE II: advancing guideline development, reporting and evaluation in health care. CMAJ 2010; 182: E839-42. (Review).

4. Drummond MF, Sculpher MJ, Claxton K, Stoddart GL, Torrance GW. Methods for the Economic Evaluation of Health Care Programmes. 4th ed. Oxford, England: Oxford University Press; 2015.

5. Sanders GD, Neumann PJ, Basu A, et al. Recommendations for conduct, methodological practices, and reporting of costeffectiveness analyses: second panel on cost-effectiveness in health and medicine. JAMA 2016; 316: 1093-103.

6. Husereau D, Drummond M, Petrou S, et al. Consolidated Health Economic Evaluation Reporting Standards (CHEERS) statement. Value Health 2013; 16: e1-5.

7. Shiroiwa T, Fukuda T, Ikeda S, Takura T, Moriwaki K. Development of an Official Guideline for the Economic Evaluation of Drugs/Medical Devices in Japan. Value Health 2017; 20: 372-8.

8. Kopec JA, Willison KD. A comparative review of four preference-weighted measures of health-related quality of life. J Clin Epidemiol 2003; 56: 317-25. (Review).

9. Drummond M, Brixner D, Gold M, Kind P, McGuire A, Nord E. Toward a consensus on the QALY. Value Health 2009; 12: S31-5.

10. Ryen L, Svensson M. The willingness to pay for a quality adjusted life year: a review of the empirical literature. Health Econ 2014. (in press).

11. Bertram MY, Lauer JA, De Joncheere $\mathrm{K}$, et al. Costeffectiveness thresholds: pros and cons. Bull World Health Organ 2016; 94: 925-30.

12. Shiroiwa T, Sung YK, Fukuda T, Lang HC, Bae SC, Tsutani K. International survey on willingness-to-pay (WTP) for one additional QALY gained: what is the threshold of cost effectiveness? Health Econ 2010; 19: 422-37.

13. Persad G, Wertheimer A, Emanuel EJ. Principles for allocation of scarce medical interventions. Lancet 2009; 373: 423-31.

14. Schlander M. The use of cost-effectiveness by the National Institute for Health and Clinical Excellence (NICE): no(t yet an) exemplar of a deliberative process. J Med Ethics 2008; 34: 5349. (Review)

15. Economic and Social Research Institute, Cabinet Office, Government of Japan. International Collaboration Projects 2010/ 2011: Studies on Labour, Tax, and Social Security through the Method of Microdata Analysis. Cost effevtiveness analyasis of dyslipidemia in japan. Accessed Aug. 4, 2017. Available at: htt p://www.esri.go.jp/jp/prj/int_prj/prj-si2010/sem_ps.html

16. Pandya A, Sy S, Cho S, Weinstein MC, Gaziano TA. Costeffectiveness of 10-year risk thresholds for initiation of statin therapy for primary prevention of cardiovascular disease. JAMA 2015; 314: 142-50.

17. Kazi DS, Moran AE, Coxson PG, et al. Cost-effectiveness of PCSK9 inhibitor therapy in patients with heterozygous familial hypercholesterolemia or atherosclerotic cardiovascular disease. JAMA 2016; 316: 743-53.

18. Annemans L, Wittrup-Jensen K, Bueno H. A review of international pharmacoeconomic models assessing the use of aspirin in 
primary prevention. J Med Econ 2010; 13: 418-27. (Review).

19. Tsutani K, Igarashi A, Fujikawa K, et al. A health economic evaluation of aspirin in the primary prevention of cardiovascular disease in Japan. Intern Med 2007; 46: 157-62.

20. Gaspoz JM, Coxson PG, Goldman PA, et al. Cost effectiveness of aspirin, clopidogrel, or both for secondary prevention of coronary heart disease. N Engl J Med 2002; 346: 1800-6.

21. Abdel-Qadir H, Roifman I, Wijeysundera HC. Cost-effectiveness of clopidogrel, prasugrel and ticagrelor for dual antiplatelet therapy after acute coronary syndrome: a decision-analytic model. CMAJ Open 2015; 3: E438-46.

22. Inoue $\mathrm{T}$, Kobayashi M, Uetsuka Y, Uchiyama S. Pharmacoeconomic analysis of cilostazol for the secondary prevention of cerebral infarction. Circ J 2006; 70: 453-8.

23. Singh SM, Wijeysundera HC. Cost-effectiveness of novel oral anticoagulants for stroke prevention in non-valvular atrial fibrillation. Curr Cardiol Rep 2015; 17: 61. (Review).

24. Kamae I, Hashimoto Y, Koretsune Y, et al. Cost-effectiveness analysis of apixaban against warfarin for stroke prevention in patients with nonvalvular atrial fibrillation in Japan. Clin Ther 2015; 37: 2837-51.

25. Cowper PA, DeLong ER, Whellan DJ, Allen LaPointe NM, Califf RM. Economic effects of beta-blocker therapy in patients with heart failure. Am J Med 2004; 116: 104-11.

26. Stewart S, McMurray JJ, Hebborn A, Coats AJ, Packer M. Carvedilol reduces the costs of medical care in severe heart failure: an economic analysis of the COPERNICUS study applied to the United Kingdom. Int J Cardiol 2005; 100: 143-9.

27. Inomata T, Izumi T, Kobayashi M. Cost-effectiveness analysis of carvedilol for the treatment of chronic heart failure in Japan. Circ J 2004; 68: 35-40.

28. Tsevat J, Duke D, Goldman L, et al. Cost-effectiveness of captopril therapy after myocardial infarction. J Am Coll Cardiol 1995; 26: 914-9.

29. Glick H, Cook J, Kinosian B, et al. Costs and effects of enalapril therapy in patients with symptomatic heart failure: an economic analysis of the Studies of Left Ventricular Dysfunction (SOLVD) Treatment Trial. J Card Fail 1995; 1: 371-80.

30. Schadlich PK, Huppertz E, Brecht JG. Cost-effectiveness analysis of ramipril in heart failure after myocardial infarction. Economic evaluation of the Acute Infarction Ramipril Efficacy (AIRE) study for Germany from the perspective of Statutory Health Insurance. Pharmacoeconomics 1998; 14: 653-69.

31. Reed SD, Friedman JY, Velazquez EJ, Gnanasakthy A, Califf RM, Schulman KA. Multinational economic evaluation of valsartan in patients with chronic heart failure: results from the Valsartan Heart Failure Trial (Val-HeFT). Am Heart J 2004; 148: $122-8$.

32. McMurray JJ, Andersson FL, Stewart S, et al. Resource utilization and costs in the Candesartan in Heart failure: Assessment of Reduction in Mortality and morbidity (CHARM) programme. Eur Heart J 2006; 27: 1447-58.

33. Imamura T, Kinugawa K, Nitta D, Komuro I. Tolvaptan reduces long-term total medical expenses and length of stay in aquaporin-defined responders. Int Heart J 2016; 57: 593-9.

34. Chen YF, Jowett S, Barton $\mathrm{P}$, et al. Clinical and costeffectiveness of epoprostenol, iloprost, bosentan, sitaxentan and sildenafil for pulmonary arterial hypertension within their licensed indications: a systematic review and economic evalu- ation. Health Technol Assess 2009; 13: 1-320. (Review).

35. Igarashi A, Inoue S, Ishii T, Tsutani K, Watanabe H. Comparative effectiveness of oral medications for pulmonary arterial hypertension network meta-analysis. Int Heart J 2016; 57: 466-72. (Review).

36. Magnuson EA, Farkouh ME, Fuster V, et al. Cost-effectiveness of percutaneous coronary intervention with drug eluting stents versus bypass surgery for patients with diabetes mellitus and multivessel coronary artery disease: results from the FREEDOM trial. Circulation 2013; 127: 820-31.

37. Cohen DJ, Osnabrugge RL, Magnuson EA, et al. Costeffectiveness of percutaneous coronary intervention with drugeluting stents versus bypass surgery for patients with 3-vessel or left main coronary artery disease: final results from the Synergy Between Percutaneous Coronary Intervention With TAXUS and Cardiac Surgery (SYNTAX) trial. Circulation 2014; 130: 114657.

38. Ariyaratne TV, Yap CH, Ademi Z, et al. A systematic review of cost-effectiveness of percutaneous coronary intervention vs. surgery for the treatment of multivessel coronary artery disease in the drug-eluting stent era. Eur Heart J Qual Care and Clin Outcomes 2016; 2: 261-70.

39. Griffin SC, Barber JA, Manca A, et al. Cost effectiveness of clinically appropriate decisions on alternative treatments for angina pectoris: prospective observational study. BMJ 2007; 334: 624.

40. Takura T, Tachibana K, Isshiki T, et al. Preliminary report on a cost-utility analysis of revascularization by percutaneous coronary intervention for ischemic heart disease. Cardiovasc Interv Ther 2017; 32: 127-36.

41. Sugimoto K, Kobayashi Y, Kuroda N, Komuro I. Cost analysis of sirolimus-eluting stents in the Japanese health insurance system. Int Heart J 2009; 50: 723-30.

42. Neyt M, Van Brabandt H, Devos C. The cost-utility of catheter ablation of atrial fibrillation: a systematic review and critical appraisal of economic evaluations. BMC Cardiovasc Disord 2013; 13: 78. (Review).

43. Kimura T, Igarashi A, Ikeda S, et al. A cost-utility analysis for catheter ablation of atrial fibrillation in combination with warfarin and dabigatran based on the CHADS (2) score in Japan. J Cardiol 69: 89-97.

44. Colquitt JL, Mendes D, Clegg AJ, et al. Implantable cardioverter defibrillators for the treatment of arrhythmias and cardiac resynchronisation therapy for the treatment of heart failure: systematic review and economic evaluation. Health Technol Assess 2014; 18: 1-560. (Review).

45. Cao C, Liou KP, Pathan FK, et al. Transcatheter aortic valve implantation versus surgical aortic valve replacement: metaanalysis of clinical outcomes and cost-effectiveness. Curr Pharm Des 2016; 22: 1965-77. (Review).

46. Sharples L, Buxton M, Caine N, et al. Evaluation of the ventricular assist device programme in the UK. Health Technol Assess 2006; 10: 1-119. (Review).

47. Seco M, Zhao DF, Byrom MJ, et al. Long-term prognosis and cost-effectiveness of left ventricular assist device as bridge to transplantation: A systematic review. Int J Cardiol 2017; 235: $22-32$ 